\title{
Capacidad innovadora de la Comunidad de Madrid a partir de las patentes concedidas entre 1996 y 2007
}

\author{
M. ${ }^{a}$ Luisa Lascurain*, M. ${ }^{a}$ Jesús Madera-Jaramillo**, \\ Eva Ortoll ${ }^{\star \star \star}$ y Elías Sanz Casado*
}

Resumen: El objetivo del presente trabajo es conocer algunas de las características de la actividad patentadora de la Comunidad de Madrid y analizar su relación con los recursos humanos y materiales que se dedican en dicha comunidad a las actividades de $\mathrm{I}+\mathrm{D}+\mathrm{i}$, así como conocer el peso de esta actividad en la CM respecto al conjunto de España. Para ello, se han analizado las patentes concedidas entre 1996 y 2007 recogidas en la base de datos INVENES de la Oficina Española de Patentes y Marcas.

Palabras clave: Innovación, Comunidad de Madrid, patentes.

\section{Capacity for innovation of the Madrid Community based on patents approved between 1996 and 2007}

Abstract: The objective of this paper is focused on analysing the patent activity of the Madrid
Community taking into account the economic and buman resources assigned to cover
its REDEI activities, as well as assessing the importance of the Madrid Community's
patenting activity compared to Spain. The analysis covers patents awarded during the
period 1996-20O7 and included in the INVENES database of the Spanish Patent and
Trade Mark Office.

Key words: Innovation, Madrid Community, patents.

\section{Introducción}

\subsection{El papel de la innovación en el desarrollo de las regiones}

El acceso al conocimiento, el talento y la creatividad son factores clave de la economía actual (Florida, 2005), puesto que la combinación de conocimiento y creatividad es una de las vías que conduce a la innovación. La innovación, jun-

* Departamento de Biblioteconomía y Documentación. Universidad Carlos III de Madrid. Correo-e: mlascura@bib.uc3m.es; elias@bib.uc3m.es.

** Centro Universitario de Investigaciones Bibliotecológicas. UNAM (México), Fundación Carolina (España). Correo-e: ramadera@yahoo.com.mx.

*** Departamento de Información y Documentación. Universitat Oberta de Catalunya. Correo-e: eortoll@uoc.edu.

Recibido: 11-11-09; 2. ${ }^{a}$ versión: 15-1-10; aceptado: 25-3-10. 
to con la competitividad, se considera uno de los principales factores de progreso y constituye una de las explicaciones básicas sobre las diferencias en el desarrollo económico y el bienestar (Grossman y Helpman, 1991; Fagerberg, 1994). De acuerdo con Camacho y Rodríguez (2005), a partir de las formulaciones iniciales de Freeman en 1987, se puede apuntar que el crecimiento económico es fundamentalmente un proceso estructural de cambio y depende en gran medida de la capacidad de las organizaciones e instituciones de reconfigurarse a ellas mismas y el territorio que ocupan.

La capacidad de innovar es función de un conjunto de factores, como el nivel de tecnología y la medida en que esta tecnología se usa, la localización geográfica, el contexto institucional, las estrategias de los actores económicos y las competencias individuales y colectivas, así como de los valores y actitudes de la base de capital humano de una región. En este sentido, la tecnología y la capacidad de innovar, así como la capacidad de asimilar las innovaciones, se consideran factores claves del desarrollo económico de cualquier territorio (Feldman y Florida, 1994; Audresch y Feldman, 1996; Furman y otros, 2002), contribuyendo positivamente al bienestar de la región (Arévalo, 2005). Precisamente tecnología e innovación son considerados como un instrumento esencial en cualquier política de desarrollo (Trajtenberg, 1990). Esta idea se reafirma por parte de la OCDE (2005) que enfatiza que los países que generan innovación crean nuevas tecnologías e impulsan la adopción de esas nuevas tecnologías para crecer más rápido que aquéllos que no lo hacen. Por tanto, hay que destacar el papel fundamental que juega la innovación y la propiedad intelectual para conseguir países más competitivos en la actual economía basada en el conocimiento.

Centrándonos en los indicadores utilizados habitualmente para medir la innovación, y siguiendo a Rodríguez-Pose y Crescenzi (2008), cabe subrayar que la investigación sobre el impacto de la innovación en el crecimiento regional en Europa ha seguido tres enfoques: modelo lineal; estudio de los sistemas de innovación regionales, y el estudio de la difusión geográfica del conocimiento. El modelo lineal considera que la investigación básica lleva a la investigación aplicada y a las invenciones. Éstas se transforman en innovaciones que a su vez originan un mayor crecimiento. Este tipo de análisis se focaliza en estudiar el vínculo entre la inversión en I+D, la capacidad de patentar y el crecimiento económico. A pesar de que hay autores que han cuestionado este modelo, para otros aún está vigente en sus planteamientos básicos, como es el caso de Balconi y otros (2009). El segundo enfoque se concentra en el estudio de los sistemas de innovación regionales y su eficiencia, básicamente se analizan los elementos territoriales - condiciones sociales y estructurales - que facilitan o frenan la generación de innovaciones. Finalmente, el enfoque sobre la difusión geográfica apunta la importancia de la proximidad física entre la transferencia y generación de nuevos conocimientos (Santos Martín y otros, 2005).

A pesar de la importancia de combinar las tres aproximaciones expuestas, el modelo lineal es útil para analizar la capacidad innovadora de una región por su 
alta capacidad explicativa: las regiones que invierten más en I+D generalmente tienden a innovar más, y crecen de forma más rápida (Rodríguez-Pose y Crescenzi, 2008).

Las patentes son uno de los indicadores más utilizados para medir la capacidad tecnológica, ello es debido a distintas razones: son fácilmente accesibles en formato electrónico, están relacionadas con el inventor, se pueden clasificar en categorías y subcategorías, identifican individuos y organizaciones, y contienen las trazas de conocimiento previo que se ha utilizado. La forma más directa de utilizar las patentes implica un recuento simple del número de patentes producidas por una organización (Nelson, 2009). Así podemos encontrar estudios que confirman la correlación entre los inputs de investigación y el número de patentes (Narin y Noma, 1987; Ahuja y Katila, 2001). Sin embargo, en otra línea de investigaciones, se considera que el recuento de patentes por sí solo es insuficiente para medir la capacidad innovadora de una región, y que la eficacia de las patentes como indicador dependerá del entorno regulador y del tipo de industria que se tenga en cuenta (Moser, 2005 citado por Nelson, 2009). A pesar de ello, distintos autores defienden que el recuento de patentes, la inversión en I+D o las citas de patentes, entre otros, se consideran medidas válidas para reflejar y evaluar la innovación en un sentido amplio, a pesar de las deficiencias o puntos débiles que se puedan asociar a cada uno de estos indicadores (Deyle y Grupp, 2005; Hagedoon y Cloddt, 2003).

\subsection{Comunidad de Madrid como unidad de análisis}

Está aceptado ampliamente que la innovación está incrustada en el territorio y que no se puede entender por completo con independencia de las condiciones institucionales y sociales en las que tiene lugar. En los últimos años se apunta la importancia de estudiar la innovación a nivel regional (Morgan y Nauwealers, 1999; Cook, 2001) ya que se ha detectado una concentración geográfica muy alta de las actividades innovadoras y la tendencia de las industrias a agruparse en espacios geográficos específicos. En este sentido existen diversas aproximaciones para considerar la unidad geográfica, desde el planteamiento de Krugman (1991) que toma como unidad geográfica de análisis el Estado, al de Audrestsch (1998) que toma como unidad geográfica de análisis la ciudad. En esta misma línea, y siguiendo a Buesa y otros (2002), en España es conveniente tomar como unidad geográfica de análisis las comunidades autónomas, debido fundamentalmente a que en la realidad española son las menores unidades administrativas que cuentan con competencias en materia de I+D.

Como se ha comentado, innovación y competitividad están obligatoriamente unidas. Uno de los indicadores más importantes de competitividad es el nivel de renta per cápita. En este sentido, el PIB de la Comunidad de Madrid desde 1955 constituye uno de los más elevados de España (Arévalo, 2005). Asimismo, en un reciente análisis sobre los sistemas de innovación en España, se constató que el

Rev. Esp. Doc. Cient., 33, 3, julio-septiembre, 458-479, 2010. ISSN: 0210-0614. doi:10.3989/redc.2010.3.761 
nivel de desarrollo de las regiones tenía un efecto positivo en los esfuerzos de $\mathrm{I}+\mathrm{D}$; de tal manera que la mayoría de las regiones con unos niveles más altos de PIB per cápita tenían a su vez un mayor nivel de gasto en I+D, siendo en el panorama español, Madrid y Cataluña las dos regiones que ocupan el lugar más destacado en innovación (Gómez y otros, 2005; Cabrer-Borrás y Serrano-Domingo, 2007). De la misma manera, Buesa y otros (2006) confirman que la Comunidad de Madrid es una de las regiones que presenta una posición más ventajosa sobre el conjunto de sistemas regionales de innovación de España.

El objetivo de este trabajo de investigación es conocer determinadas características de la capacidad innovadora de la Comunidad de Madrid, como son el número y la evolución temporal de las patentes concedidas, así como las instituciones y temas con mayor actividad y atracción inventora. Todo ello, se comparará con las inversiones en I+D que se realizan en la Comunidad de Madrid, y los recursos humanos especializados que se dedican a las actividades de investigación en la región.

\section{Material y métodos}

Para la obtención de los datos relativos a las invenciones se utilizó la sección Interpat de la base de datos INVENES producida por la Oficina Española de Patentes y Marcas (OEPM), delimitando el período temporal a los años comprendidos entre 1996 y 2007.

Se consideraron todas las concesiones españolas con la delimitación temporal correspondiente al período de estudio, descargándose con este criterio de búsqueda un total de 20.433 patentes. Para el manejo de los datos se creó una base de datos relacional, administrada por el gestor de bases de datos MySQL. También, se desarrollaron scripts con el lenguaje de programación Perl, que permitieron ingresar a la base de datos los registros recuperados.

Las variables consideradas fueron: año de concesión, nombre de los solicitantes, clasificación internacional de patentes (a nivel tanto de secciones como de clases), sectores institucionales y provincia del solicitante para delimitar el análisis a la CM.

Tras la normalización de los registros, los solicitantes se clasificaron en seis sectores institucionales asignando el valor completo de la invención a los sectores participantes en cada uno de los registros procesados. Estos sectores son las empresas, categoría que se adscribió a aquellos solicitantes que en su denominación incluían alguna indicación para ser consideradas como tales (por ejemplo, SL, SA...), los particulares, las universidades, el CSIC (que se analizó por separado de otros OPI por su peso), otros OPI y otros sectores. Se empleó la Clasificación Internacional de Patentes (CIP) para el análisis temático, que se realizó a nivel de sección y de clase. En este caso también se adjudicó la clasificación mediante la técnica de recuento completo a cada una de las secciones o clases de los registros. 
Para el tratamiento de los datos se procedió a la elaboración de tablas de contingencia y a la obtención de indicadores unidimensionales relativos a producción tecnológica, evolución anual de la producción, sectores institucionales y temáticas, así como a los indicadores multidimensionales. En este caso, se empleó el Análisis de Correspondencias (AC) para relacionar las fechas de concesión y los sectores, y estos últimos con las temáticas. Este análisis fue realizado con el programa estadístico Xlstat.

Para recoger los datos estadísticos sobre gastos en I+D y sobre investigadores por sectores tanto en España como en la CM se utilizó INEbase, sistema del Instituto Nacional de Estadística (INE) para almacenar la información en Internet.

\section{Resultados y discusión}

\subsection{Situación de la CM con respecto al conjunto español en gastos en I+D y en personal dedicado a la investigación.}

La CM ha ejecutado una parte muy importante del gasto de las actividades de I+D respecto al conjunto de España, con un porcentaje promedio del 29,90\% del total de España en el período estudiado. Este dato da una idea de la dimensión que tiene la CM dentro del país en este tipo de actividades (tabla I).

Cuando se analiza por sectores de ejecución, se advierte que el gasto de I+D correspondiente al conjunto de España muestra diferencias importantes respecto al observado en la CM. Por ejemplo, el ejecutado por la Administración Pública fue en promedio el 16,54\%, casi 10 puntos inferior al de la CM (25,66\%), mientras que el gasto ejecutado en la Enseñanza Superior fue del 29,29\%; es decir, más de 12 puntos por encima del correspondiente a la CM (16,98\% para este mismo sector). Por su parte, las empresas e Instituciones Privadas sin Fines de Lucro (IPSFL), con un porcentaje de gasto para el conjunto del España del 54,16\%, mostraron unos valores inferiores en algo más de 3 puntos a los de la CM (57,36\%).

En 2007 el gasto destinado a I+D en España alcanzó el 1,27\% del PIB, que supone la mitad de lo que destinan los países de la OCDE, mientras que el gasto correspondiente de la $\mathrm{CM}$ en ese mismo año ha sido en promedio notablemente superior (1,93\%) al del conjunto de España (INEbase, 2009).

Al considerar el incremento anual medio de gasto ejecutado en todo el período estudiado (tabla II), encontramos que éste es inferior en la CM (10,79\%) que en el conjunto español (12,03\%). También es posible observar una importante diferencia cuando analizamos el incremento del gasto en el año 2007 respecto al 1997, puesto que éste fue del 230,35\% en España, mientras que en la CM este porcentaje fue notablemente inferior $(175,47 \%)$.

Los recursos humanos dedicados a la investigación constituyen otra de las piezas fundamentales para el desarrollo de las actividades científicas y tecnológicas en los países desarrollados. En la tabla III se muestra la evolución de los recursos humanos, durante todo el período de estudio, tanto en el conjunto de España como en la CM. En este sentido, hay que señalar que sólo se han considerado en

Rev. Esp. Doc. Cient., 33, 3, julio-septiembre, 458-479, 2010. ISSN: 0210-0614. doi:10.3989/redc.2010.3.761 


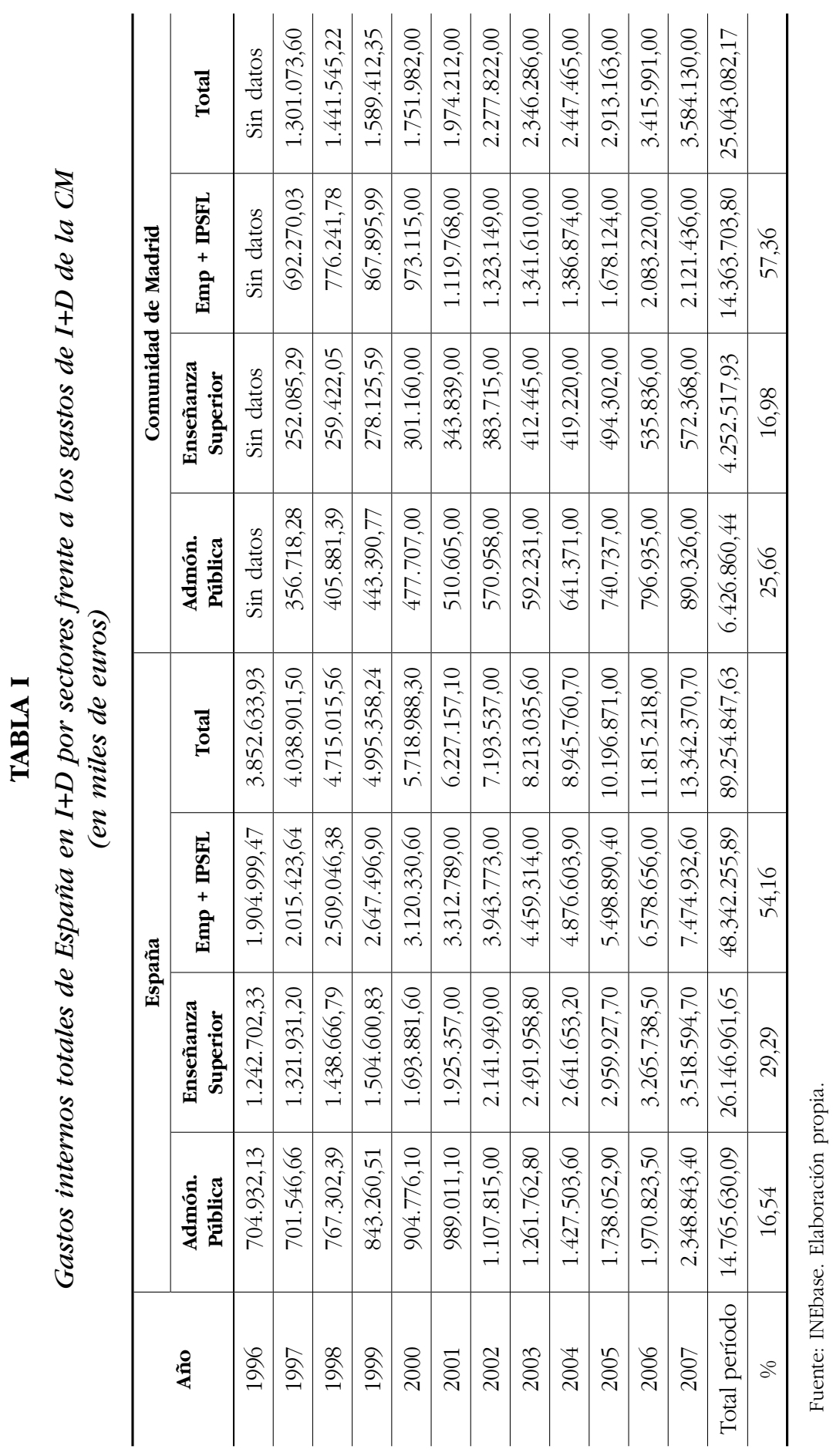


TABLA II

Gastos internos totales de España y de la CM en I+D. Incrementos anuales

\begin{tabular}{|c|c|c|c|c|c|c|}
\hline \multirow{2}{*}{ Año } & \multicolumn{2}{|c|}{ Admón. Pública } & \multicolumn{2}{|c|}{ Enseñanza Superior } & \multicolumn{2}{|c|}{ Empresas + IPSFL } \\
\hline & España & $\mathbf{C M}$ & España & $\mathbf{C M}$ & España & $\mathbf{C M}$ \\
\hline 1996 & & & & & & \\
\hline 1997 & $-0,48$ & & 6,38 & & 5,80 & \\
\hline 1998 & 9,37 & 13,78 & 8,83 & 2,91 & 24,49 & 12,13 \\
\hline 1999 & 9,90 & 9,24 & 4,58 & 7,21 & 5,52 & 11,81 \\
\hline 2000 & 7,29 & 7,74 & 12,58 & 8,28 & 17,86 & 12,12 \\
\hline 2001 & 9,31 & 6,89 & 13,67 & 14,17 & 6,17 & 15,07 \\
\hline 2002 & 12,01 & 11,82 & 11,25 & 11,60 & 19,05 & 18,16 \\
\hline 2003 & 13,90 & 3,73 & 16,34 & 7,49 & 13,07 & 1,40 \\
\hline 2004 & 13,14 & 8,30 & 6,01 & 1,64 & 9,36 & 3,37 \\
\hline 2005 & 21,75 & 15,49 & 12,05 & 17,91 & 12,76 & 21,00 \\
\hline 2006 & 13,39 & 7,59 & 10,33 & 8,40 & 19,64 & 24,14 \\
\hline 2007 & 19,18 & 11,72 & 7,74 & 6,82 & 13,62 & 1,83 \\
\hline Total* & 11,71 & 9,63 & 9,98 & 8,64 & 13,39 & 12,1 \\
\hline
\end{tabular}

* Valores promedio del crecimiento anual.

Fuente: INEbase. Elaboración propia.

este estudio los investigadores en equivalencia a jornada completa (EJC), según la clasificación que realiza el INE, dejando fuera del análisis a otro personal de I+D, como técnicos y auxiliares. La tabla III permite comparar los investigadores adscritos a los distintos sectores en el conjunto de España y en la CM.

La CM en promedio cuenta con el $24 \%$ de los investigadores a jornada completa (EJC) del conjunto nacional. En cuanto a la distribución por sectores, se aprecian diferencias importantes entre los valores promedio observados en España y en la Comunidad de Madrid. El sector correspondiente a Enseñanza Superior es el que presenta diferencias más elevadas ya que son casi 19 puntos los que separan a la CM $(34,11 \%)$ del conjunto de España $(52,85 \%)$. En el caso del porcentaje correspondiente a personal investigador que trabaja en la Administración Pública, el valor más alto lo presenta la CM (29,63\%) respecto al conjunto de España (17,41\%). Esto puede ser debido al efecto capitalidad, que favoreció la creación de un elevado número de instituciones científicas en la región madrileña, y, posteriormente, con las transferencias de competencias de la gestión de la investigación, pasaron a depender directamente de la administración autonómica.

En cuanto a las empresas e IPSFL, también es superior el porcentaje de investigadores dedicado a I+D en la CM (36,26\%) que en el conjunto de España $(29,74 \%)$. Esto también puede estar vinculado con el efecto capitalidad que ha hecho que muchas empresas se establezcan en la región madrileña. 


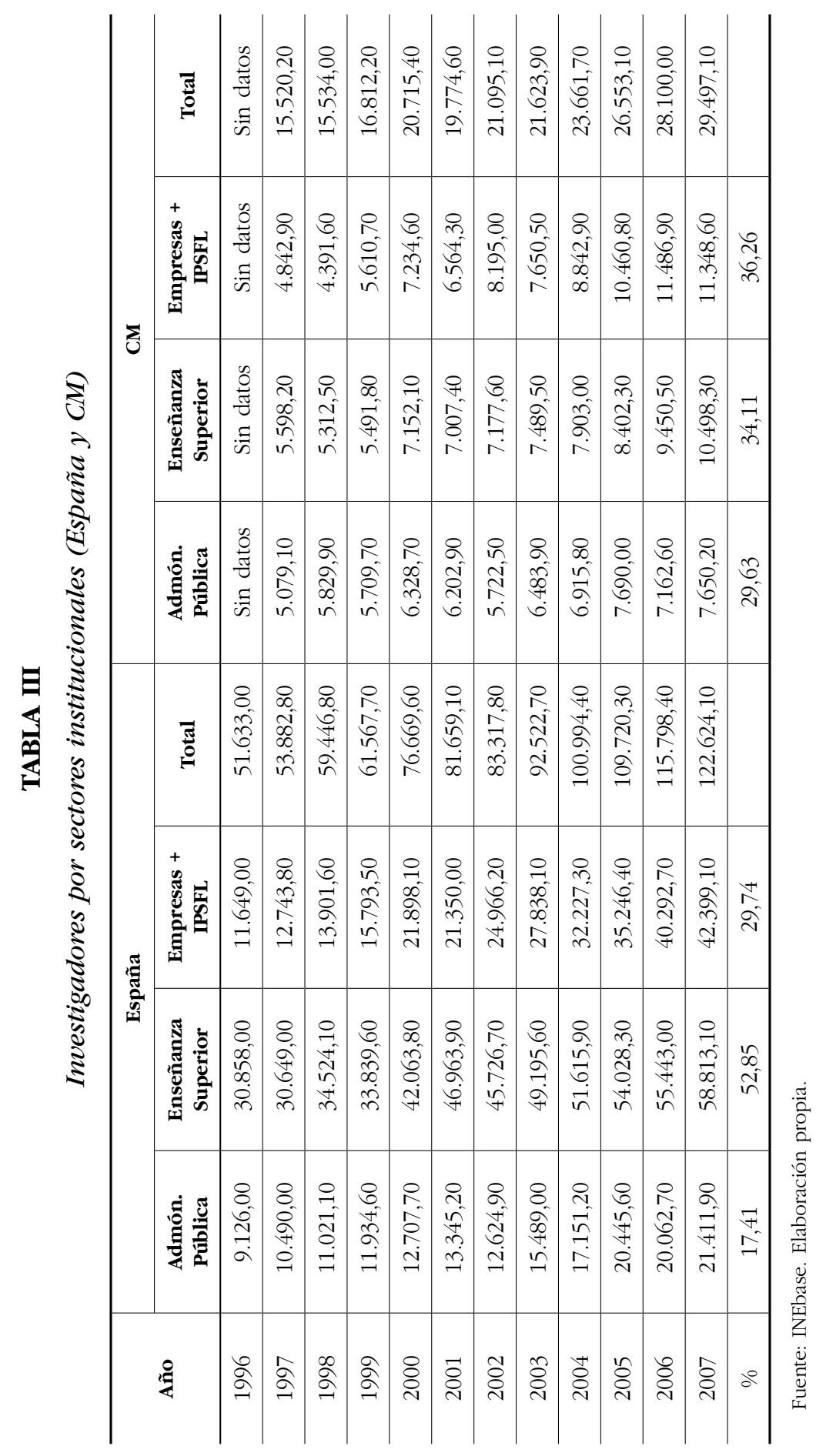


$\mathrm{Al}$ analizar el incremento anual promedio de investigadores en actividades de I+D, se advierte un mayor crecimiento en el conjunto de España (8,33\%) que en la CM (6,86\%) (tabla IV). Del mismo modo, el incremento porcentual en el número de investigadores incorporados al sistema científico en 2007 respecto a 1997 es también notablemente superior en España (127,58\%) que en la CM (90,06\%) (tabla III).

En el ámbito de los recursos humanos dedicados a I+D (número de investigadores por cada 1.000 habitantes), España se encuentra alejada de la media de la OCDE (5,2 investigadores por cada 1.000 habitantes frente a 8 investigadores por 1.000 habitantes de la OCDE). Sin embargo, hay que destacar el esfuerzo que está realizando España para incrementar estas cifras, ya que ha experimentado un crecimiento de universitarios más rápido que en el resto de la OCDE (Comisión Interministerial de Ciencia y Tecnología, 2007).

\section{TABLA IV}

Investigadores por sectores institucionales (España y CM). Incremento anual

\begin{tabular}{|c|c|c|c|c|c|c|}
\hline \multirow{2}{*}{ Año } & \multicolumn{2}{|c|}{ Admón. Pública } & \multicolumn{2}{|c|}{ Enseñanza Superior } & \multicolumn{2}{|c|}{ Empresas + IPSFL } \\
\hline & España & CM & España & CM & España & $\mathbf{C M}$ \\
\hline \multicolumn{7}{|l|}{1996} \\
\hline 1997 & 14,95 & & $-0,68$ & & 9,40 & \\
\hline 1998 & 5,06 & 14,78 & 12,64 & $-5,10$ & 9,09 & $-9,32$ \\
\hline 1999 & 8,29 & $-2,06$ & $-1,98$ & 3,38 & 13,61 & 27,76 \\
\hline 2000 & 6,48 & 10,84 & 24,30 & 30,23 & 38,65 & 28,94 \\
\hline 2001 & 5,02 & $-1,99$ & 11,65 & $-2,02$ & $-2,50$ & $-9,27$ \\
\hline 2002 & $-5,40$ & $-7,74$ & $-2,63$ & 2,43 & 16,94 & 24,84 \\
\hline 2003 & 22,69 & 13,31 & 7,59 & 4,35 & 11,50 & $-6,64$ \\
\hline 2004 & 10,73 & 6,66 & 4,92 & 5,52 & 15,77 & 15,59 \\
\hline 2005 & 19,21 & 11,19 & 4,67 & 6,32 & 9,37 & 18,30 \\
\hline 2006 & $-1,87$ & $-6,86$ & 2,62 & 12,48 & 14,32 & 9,81 \\
\hline 2007 & 6,72 & 6,81 & 6,08 & 11,09 & 5,23 & $-1,20$ \\
\hline Total* & 8,35 & 4,49 & 6,29 & 6,87 & 12,85 & 9,88 \\
\hline
\end{tabular}

* Valores promedio del crecimiento anual.

Fuente: INEbase. Elaboración propia.

\subsection{La invención en la $\mathrm{CM}$ y en el conjunto de España}

Durante el período analizado, el número total de patentes concedidas en España ha tenido un incremento medio anual del $17,22 \%$, mientras que el observado en la CM fue inferior, concretamente del 11,72\% (tabla V). Estos datos son 
llamativos ya que muestran una menor actividad patentadora por parte de la CM con respecto al conjunto español en los últimos años. Hay que señalar aquí que esto coincide con los incrementos anuales promedio tanto en gasto como en investigadores a jornada completa, inferiores en la CM que en el conjunto de España.

El análisis del número de patentes concedidas en España y en la CM a lo largo del período de estudio muestra que la región madrileña ha sido responsable del 20,74\% de las concedidas en el conjunto del país. En este sentido, es importante ver la evolución de la contribución de la CM al conjunto español, puesto que esta comienza representando el 25,32\% de total en 1996, para descender al 16,30\% en 2007. Evidentemente esto podría deberse al fuerte incremento de las patentes concedidas al resto de comunidades autónomas, que hace que la CM pierda peso en el conjunto nacional. Sin embargo, el menor ritmo de crecimiento anual en la CM, tal y como se acaba de mencionar, y su coincidencia con la disminución en las inversiones en recursos económicos y humanos, hace pensar que las causas estén más vinculadas con una menor inversión de recursos en la región madrileña.

TABLA V

Número de patentes concedidas en España y en la CM

\begin{tabular}{c|c|c|c|c|c}
\hline Año & $\begin{array}{c}\text { Patentes } \\
\text { España }\end{array}$ & $\begin{array}{c}\text { Incremento } \\
\text { anual }\end{array}$ & Patentes CM & $\begin{array}{c}\text { Incremento } \\
\text { anual (\%) }\end{array}$ & $\begin{array}{c}\text { CM respecto } \\
\text { a España (\%) }\end{array}$ \\
\hline 1996 & 778 & & 197 & & 25,32 \\
\hline 1997 & 1.201 & 54,37 & 284 & 44,16 & 23,65 \\
\hline 1998 & 1.762 & 46,71 & 399 & 40,49 & 22,64 \\
\hline 1999 & 1.838 & 4,31 & 412 & 3,26 & 22,42 \\
\hline 2000 & 1.610 & $-12,40$ & 372 & $-9,71$ & 23,11 \\
\hline 2001 & 1.774 & 10,19 & 382 & 2,69 & 21,53 \\
\hline 2002 & 921 & $-48,08$ & 190 & $-50,26$ & 20,63 \\
\hline 2003 & 1.803 & 95,77 & 337 & 77,37 & 18,69 \\
\hline 2004 & 1.860 & 3,16 & 366 & 8,61 & 19,68 \\
\hline 2005 & 2.380 & 27,96 & 412 & 12,57 & 17,31 \\
\hline 2006 & 2.028 & $-14,79$ & 358 & $-13,11$ & 17,65 \\
\hline 2007 & 2.478 & 22,19 & 404 & 12,85 & 16,30 \\
\hline Total & 20.433 & & 4.113 & & 20,74 \\
\hline Promedio & $1.702,75$ & 17,22 & 342,75 & 11,72 & \\
\hline
\end{tabular}

Fuente: OEPM. Elaboración propia. 


\subsection{Características de la invención en la CM}

La tabla VI muestra la evolución de las patentes en la CM según los sectores institucionales contemplados en este análisis. Dicha asignación por sectores tiene como limitación el que los derechos de las patentes surgidos en el seno de una determinada institución (por ejemplo en una universidad) pueden ser solicitados por personas físicas o jurídicas ajenas a ésta.

Como era de esperar, las empresas y los particulares son los sectores que acumulan un mayor número de patentes a lo largo de los 12 años de estudio.

\section{TABLA VI}

Evolución de patentes en la CM según sectores institucionales e incrementos anuales (entre paréntesis)

\begin{tabular}{c|c|c|c|c|c|c}
\hline Año & Empresas & Particulares & CSIC & Universidad & OPIS & Otros \\
\hline 1996 & 103 & 60 & 20 & 9 & 5 & 4 \\
\hline \multirow{2}{*}{1997} & 156 & 82 & 25 & 20 & 6 & 3 \\
& $(51,46)$ & $(3,67)$ & $(25,00)$ & $(122,22)$ & $(20,00)$ & $(-25,00)$ \\
\hline \multirow{2}{*}{1998} & 181 & 155 & 24 & 34 & 5 & 10 \\
& $(16,03)$ & $(8,90)$ & $(-4,00)$ & $(70,00)$ & $(-16,67)$ & $(233,33)$ \\
\hline \multirow{2}{*}{1999} & 181 & 148 & 56 & 32 & 6 & 11 \\
& $(0)$ & $(-0,45)$ & $(133,33)$ & $(-5,88)$ & $(20,00)$ & $(10,00)$ \\
\hline \multirow{2}{*}{2000} & 179 & 114 & 58 & 25 & 5 & 7 \\
& $(-1,10)$ & $(-2,30)$ & $(3,57)$ & $(-21,88)$ & $(-16,67)$ & $(-36,36)$ \\
\hline \multirow{2}{*}{2001} & 168 & 136 & 36 & 54 & 6 & 5 \\
& $(-6,15)$ & $(1,93)$ & $(-37,93)$ & $(116,00)$ & $(20,00)$ & $(-28,57)$ \\
\hline \multirow{2}{*}{2002} & 65 & 62 & 41 & 36 & 2 & 4 \\
& $(-61,31)$ & $(-5,44)$ & $(13,89)$ & $(-33,33)$ & $(-66,67)$ & $(-20,00)$ \\
\hline \multirow{2}{*}{2003} & 141 & 105 & 55 & 47 & 8 & 4 \\
& 116,92 & 6,94 & 34,15 & 30,56 & 300,00 & 0,00 \\
\hline \multirow{2}{*}{2004} & 158 & 97 & 78 & 48 & 9 & 7 \\
& $(12,06)$ & $(-0,76)$ & $(41,82)$ & $(2,13)$ & $(12,50)$ & $(75,00)$ \\
\hline \multirow{2}{*}{2005} & 164 & 136 & 90 & 41 & 10 & 9 \\
& $(3,80)$ & $(4,02)$ & $(15,38)$ & $(-14,58)$ & $(11,11)$ & $(28,57)$ \\
\hline \multirow{2}{*}{2006} & 144 & 101 & 62 & 63 & 11 & 17 \\
& $(-12,20)$ & $(-2,57)$ & $(-31,11)$ & $(53,66)$ & $(10,00)$ & $(88,89)$ \\
\hline \multirow{2}{*}{2007} & 157 & 127 & 71 & 58 & 14 & 12 \\
& $(9,03)$ & $(2,57)$ & $(14,52)$ & $(-7,94)$ & $(27,27)$ & $(-29,41)$ \\
\hline & 1797 & 1323 & 616 & 467 & 87 & 93 \\
& $(11,68)^{*}$ & $(1,50)^{*}$ & $(18,97)^{*}$ & $(28,27)^{*}$ & $(29,17)^{*}$ & $(26,95)^{*}$ \\
\hline
\end{tabular}

* Promedios anuales.

Fuente: OEPM. Elaboración propia. 
En valores porcentuales ambos sectores suman el 71,18\% del total, mientras que las instituciones más ligadas a la investigación, como son las universidades, CSIC y otros OPI acumulan el 26,69\%. Sin embargo, hay que destacar el gran incremento medio anual de las patentes concedidas a estos sectores a lo largo del período estudiado. Los otros OPI, presentan un incremento medio anual del $29,17 \%$, las universidades del 28,27\% y CSIC del 18,97\%. Estos importantes aumentos, aunque partiendo de valores bajos, vendrían a evidenciar el interés cada vez mayor que tienen los sectores mencionados por la generación de este tipo de conocimiento. Con respecto al CSIC, ha de tenerse en cuenta que el total de las patentes se gestiona desde la organización central de Madrid, aunque pueda proceder de institutos de otras Comunidades Autónomas.

En cuanto al sector empresarial, el promedio de variación anual se incrementa en el 11,68\%. Este valor se encuentra en consonancia con el incremento promedio anual de gasto de I+D ejecutado por este sector en la CM que ha sido del $12,04 \%$ en el período considerado. También es preciso señalar el cambio de tendencia que se observa entre el sector empresarial y las universidades, el CSIC o los otros OPI, que han incrementado notablemente su actividad patentadora. Una posible explicación a esta situación podría darse por el importante aumento de la cooperación que se está produciendo entre las empresas y los centros públicos de investigación, sobre todo al aumentar la complejidad tecnológica de la innovación, tal y como se reconoce en el trabajo de Fernández de Lucio y otros (1983).

A partir de los números índice, en los que se ha tomado como base el primer año de estudio (1996), se ha podido comprobar cómo las universidades, pese a su escasa presencia en valores absolutos al inicio del período, constituyen el sector que presenta un mayor crecimiento, que alcanza en el año 2006 el 722,22\%. El CSIC, sector también vinculado a la investigación, experimenta asimismo crecimientos importantes con respecto al año base, como el del $450 \%$ para el año 2005. En cuanto a otros OPI, los valores obtenidos oscilan entre un crecimiento del 280\% en el último año del estudio al decremento del 40\% en 2002 y el estacionamiento de los años 1998 y 2000. Las empresas presentan un crecimiento que no supera el 175\% (años 1998 y 1999) y un año, el 2002, con un decremento del $63,11 \%$. Las patentes concedidas anualmente a los particulares crecen a lo largo del período de estudio, con un máximo del 258,33\% en el año 1998. Los datos se muestran en la figura 1.

En la figura 2 se muestra la evolución de la actividad patentadora de los distintos sectores institucionales de la Comunidad de Madrid a lo largo de los años descritos. Para ello se ha realizado un Análisis de Correspondencias (AC) que se ha representado mediante un gráfico de burbujas. El tamaño de las burbujas en el caso de la variable "sector" es indicativo de la proporción de patentes concedidas en las categorías de la misma. La posición en el mapa informa sobre los perfiles evolutivos que muestra cada uno de los sectores. En este sentido, tanto empresas como particulares que se sitúan próximos a la zona central del mapa presentan un perfil similar a lo largo de los años estudiados, mientras que las 


\section{FIGURA 1}

Incremento del número de patentes concedidas por sectores (números índice. Año base 1996)

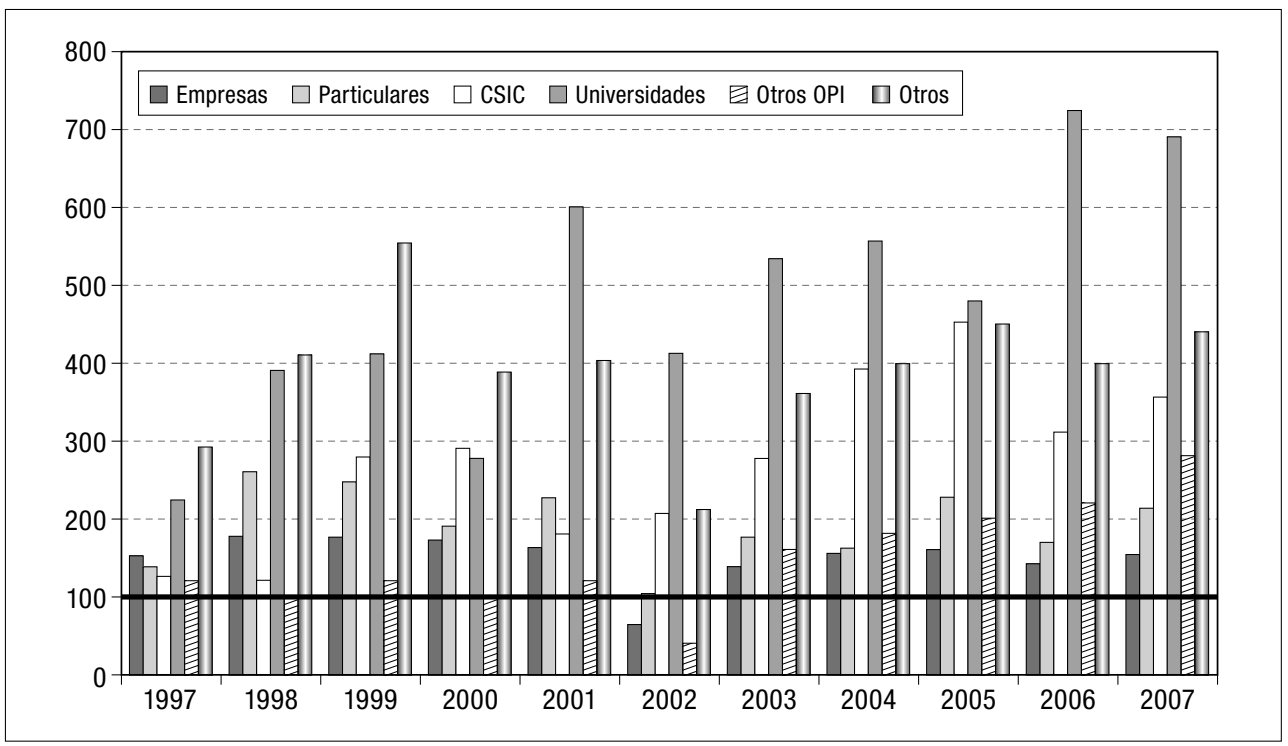

FIGURA 2

Evolución de la actividad patentadora de los sectores (análisis de correspondencias)

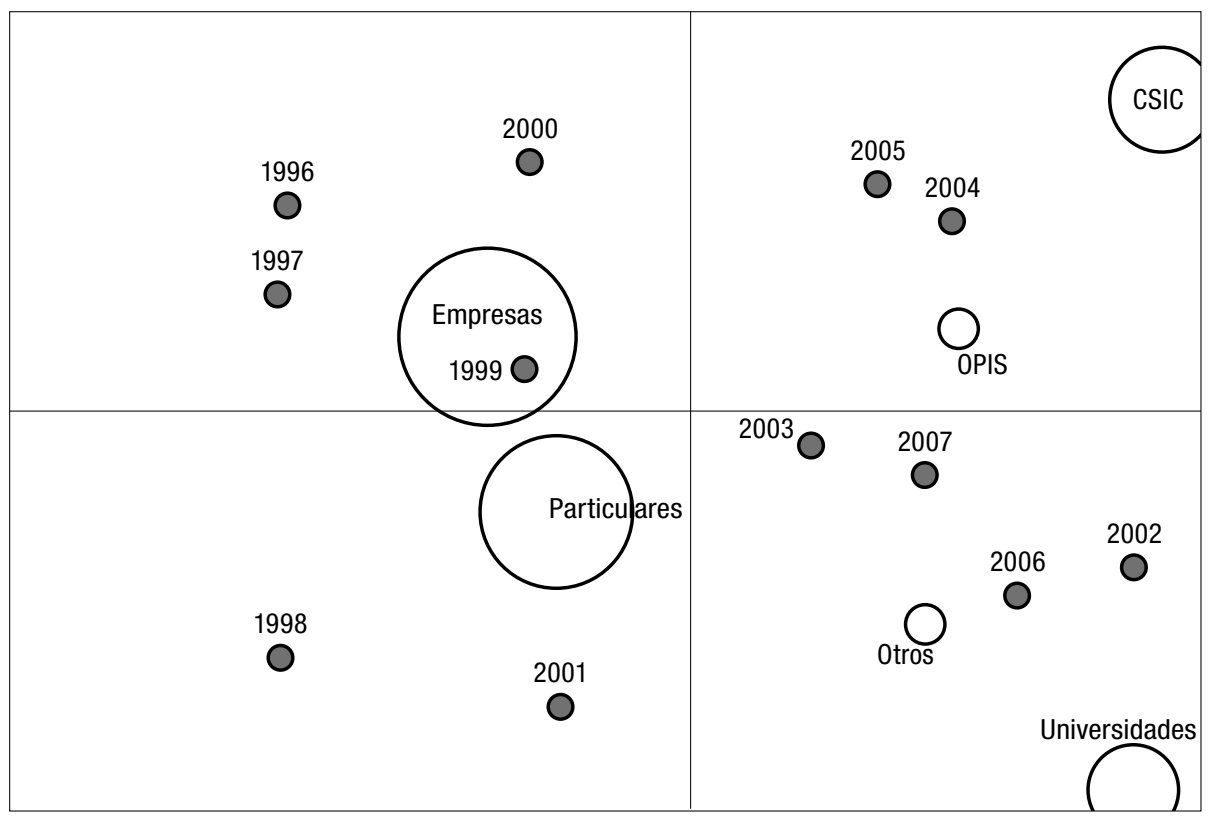


universidades, en el cuadrante inferior derecho del mapa junto con "Otros", concentra su actividad en los últimos años del estudio (2006 y 2007). Por otro lado, el CSIC y otros OPI (cuadrante superior derecho) tienen una mayor actividad en los años 2004 y 2005.

Otro de los aspectos que se ha querido conocer en este trabajo es la temática en la que se patenta en la Comunidad de Madrid. En este sentido, y para determinar las categorías de esta variable, se ha utilizado la Clasificación Internacional de Patentes (CIP), incluyéndose todos los códigos de clasificación asignados a cada patente.

En la tabla VII se muestran los datos correspondientes a la asignación de las ocho secciones del CIP. La sección A, "Necesidades de la Vida" que incluye las subsecciones de Actividades rurales; Alimentación y Tabaco; Objetos personales o domésticos; Salud, Protección y Farmacia, recoge el 23,56\% de las patentes. Le sigue, con un porcentaje similar (el 23,36\%) la sección C, "Química/Metal". Sólo estas dos secciones abarcan casi la mitad de las patentes concedidas en todo el período considerado (el 46,92\%).

La clasificación de "Física" (G), que incluye Instrumentos y Ciencia Nuclear, ocupa el tercer lugar con un porcentaje del 22,34\%.

"Técnicas industriales y transportes" (B), clasificación que abarca los temas de Separación y Mezcla, Conformación Mecánica; Imprenta; Transportes; Almacenamiento; Tecnología de las microestructuras y Nanotecnología, ocupa el cuarto lugar con el 22,04\%. "Electricidad" (H) representa el 13,90\%. Las siguientes temáticas no llegan al 10\% de las invenciones cada una de ellas, como son: "Construcción" (E) (Edificios y perforación del suelo y explotación minera) que ocupa el sexto lugar con el 9,36\%, "Mecánica" (F) con el 7,98\%, y la temática "Textiles y Papel" (D) se limita a una aportación del 1,01\% del total.

\section{TABLA VII}

Temática de las patentes concedidas a la CM

\begin{tabular}{l|c|c}
\multicolumn{1}{c|}{ Secciones } & Frecuencia & $\mathbf{\%}$ \\
\hline A: Necesidades de la vida & 1.022 & 23,56 \\
\hline C: Química/Metal & 1.013 & 23,36 \\
\hline G: Física & 969 & 22,34 \\
\hline B: Técnicas industria/Transportes & 956 & 22,04 \\
\hline H: Electricidad & 603 & 13,90 \\
\hline E: Construcción & 406 & 9,36 \\
\hline F: Mecánica & 346 & 7,98 \\
\hline D: Textiles/Papel & 44 & 1,01 \\
\hline Total & 4.337 & \\
\hline
\end{tabular}

Fuente: OEPM. Elaboración propia. 
La evolución de las secciones a lo largo del período analizado se muestra en la figura 3, y ha sido obtenida mediante un análisis de correspondencias. Los temas tratados que tienen una frecuencia similar en todos los años analizados son: Necesidades de la Vida, Técnicas industria/Transportes, Construcción, Química/Metalurgia y Física. Respecto a las otras temáticas, es interesante señalar los casos de Textiles/Papel y Electricidad, que se sitúan en el cuadrante superior e inferior derecho del mapa, debido a que su actividad patentadora fue más activa a finales de la década de los noventa.

\section{FIGURA 3}

Evolución temporal de los temas en los que se patenta en la CM (análisis de correspondencias)

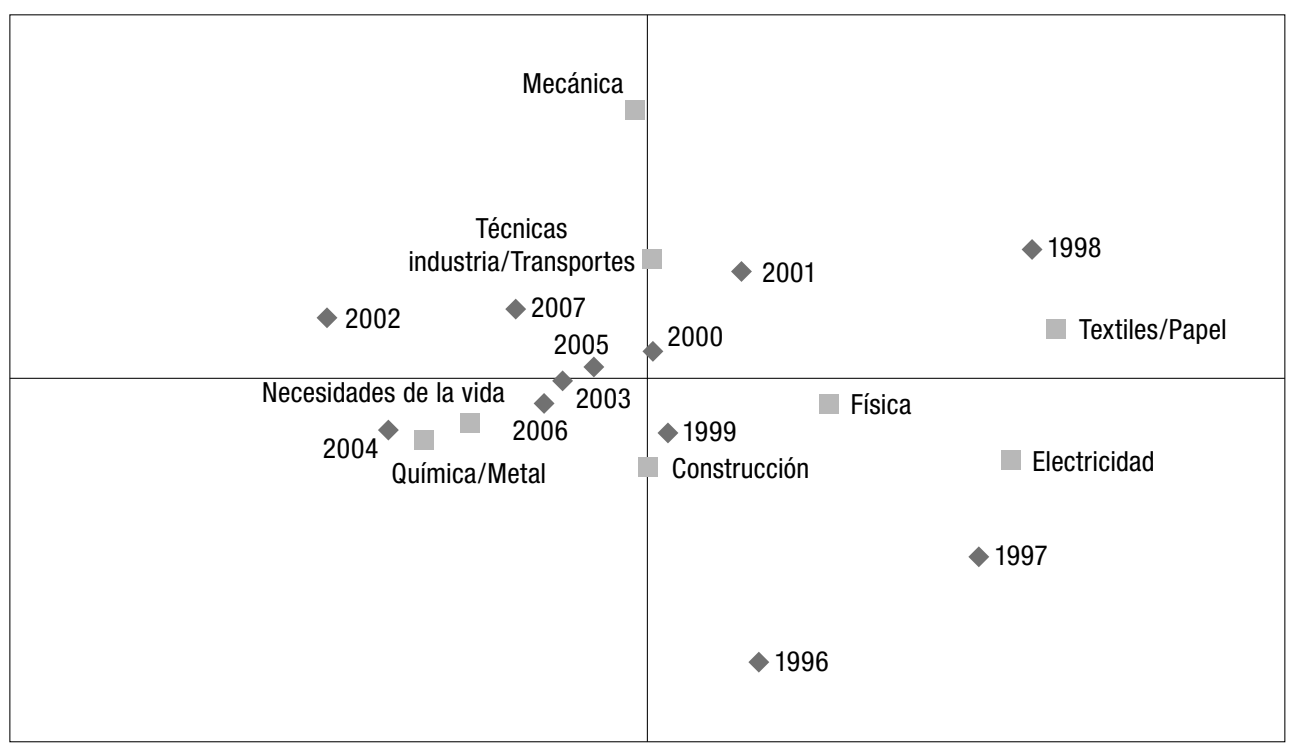

Con el fin de establecer la relación entre temas en que se patenta y los distintos sectores de la CM, se ha realizado un análisis de correspondencias. La representación gráfica obtenida (figura 4) permite observar la diferenciación en la forma de agruparse los sectores institucionales en torno a las temáticas sobre las que versan sus patentes. Las instituciones con mayor actividad investigadora (universidades, CSIC y otros OPI) tienen un perfil más específico que el resto, y que gira en torno a los temas agrupados en la clasificación de Química/Metalurgia. Mientras que los sectores que más patentan (empresas y particulares) se sitúan próximos al resto de las temáticas, lo que indicaría un perfil más difuso.

En la figura 4 llama la atención el caso de la Física que aparece en el mapa en la posición central muy vinculada al mundo empresarial, pero en el mismo 
cuadrante y también cercana a las universidades, debido a la importante actividad de estas instituciones académicas en relación con los temas de esta sección.

\section{FIGURA 4}

Análisis de correspondencias. Temas (a nivel sección) vs sectores

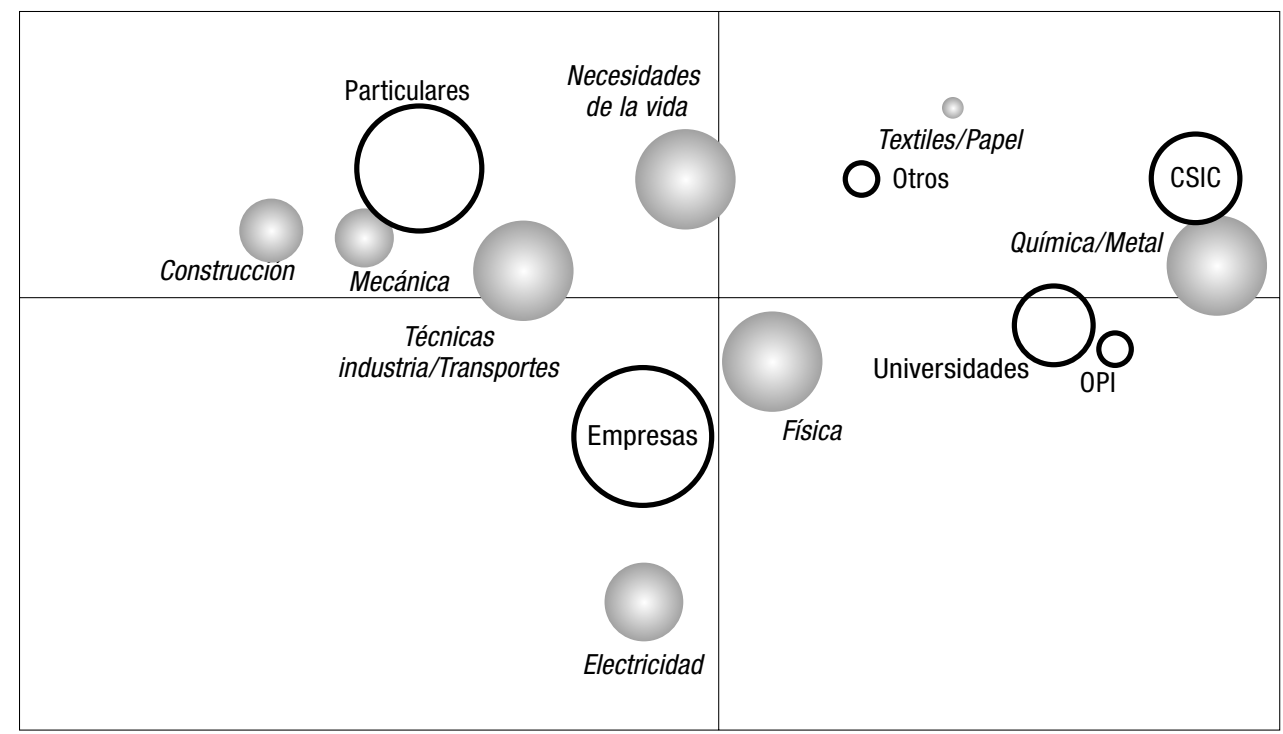

Una información más detallada sobre los temas de mayor interés a la hora de patentar se obtiene a partir del análisis de las clases de la clasificación CIP con más patentes concedidas.

Con el fin de facilitar la visualización de los datos sólo se han considerado aquellos temas en los que aparecía un número de patentes igual o superior a 30 (figura 5). En este mapa no figuran los otros OPI ya que no cumplen el requisito establecido en el umbral.

Nuevamente las universidades y el CSIC aparecen próximas en el mismo cuadrante y con un perfil diferenciado con respecto al resto de sectores institucionales, situándose próximos a temas en los que patentan, y que varios de ellos están relacionados con la Química, como son: C12 (Química orgánica), C7 (Bioquímica; Cerveza; Bebidas alcohólicas; Vino; Vinagre; Microbiología; Enzimología; Técnicas de mutación o de Genética), C01 (Química inorgánica), C04 (Cementos, hormigón, piedra artificial, cerámicas y refractarios) y B01 (Procedimientos o aparatos físicos o químicos en general). Algunos de estos están vinculados también con la Física (G01, Metrología).

Algo más próximos a la zona central del mapa se sitúan los particulares y empresas que muestran de nuevo un perfil amplio y difuso, ya que en su entorno se sitúan temas procedentes de distintas secciones. En el cuadrante superior 
izquierdo aparecen los temas más ligados a la actividad patentadora de los particulares, como B60 (Vehículos en general), E04 (Edificios), o B65 (Transporte; embalaje; almacenado; manipulación de materiales delgados o filiformes).

En la zona central del mapa se agruparían las clases en las que más patentan todas las instituciones analizadas. En este estudio, las tres clases que se sitúan en esta zona son las incluidas en la sección A (Necesidades de la Vida), todas ellas con un gran número de patentes asociadas. Se trata de Ciencias Médicas o Veterinarias e Higiene (A61), Agricultura (A01) y Alimentos (A23).

En la zona inferior izquierda se sitúa otro grupo de temas, en este caso vinculados a las patentes procedentes del ámbito empresarial, en el que destacan los dispositivos de control (G07) y Técnica de las comunicaciones eléctricas (H04).

Finalmente, como puede observarse en la figura 5, aparecen 2 perfiles bien diferenciados, por un lado las universidades y el CSIC patentando en temas con un mayor componente investigador, y por otro lado las empresas y particulares con un perfil que implica, en la mayoría de los casos, investigación aplicada.

\section{FIGURA 5}

Análisis de correspondencias. Temas (nivel 3) vs sectores (sólo se ban representado los temas que aparecen al menos en 30 registros)

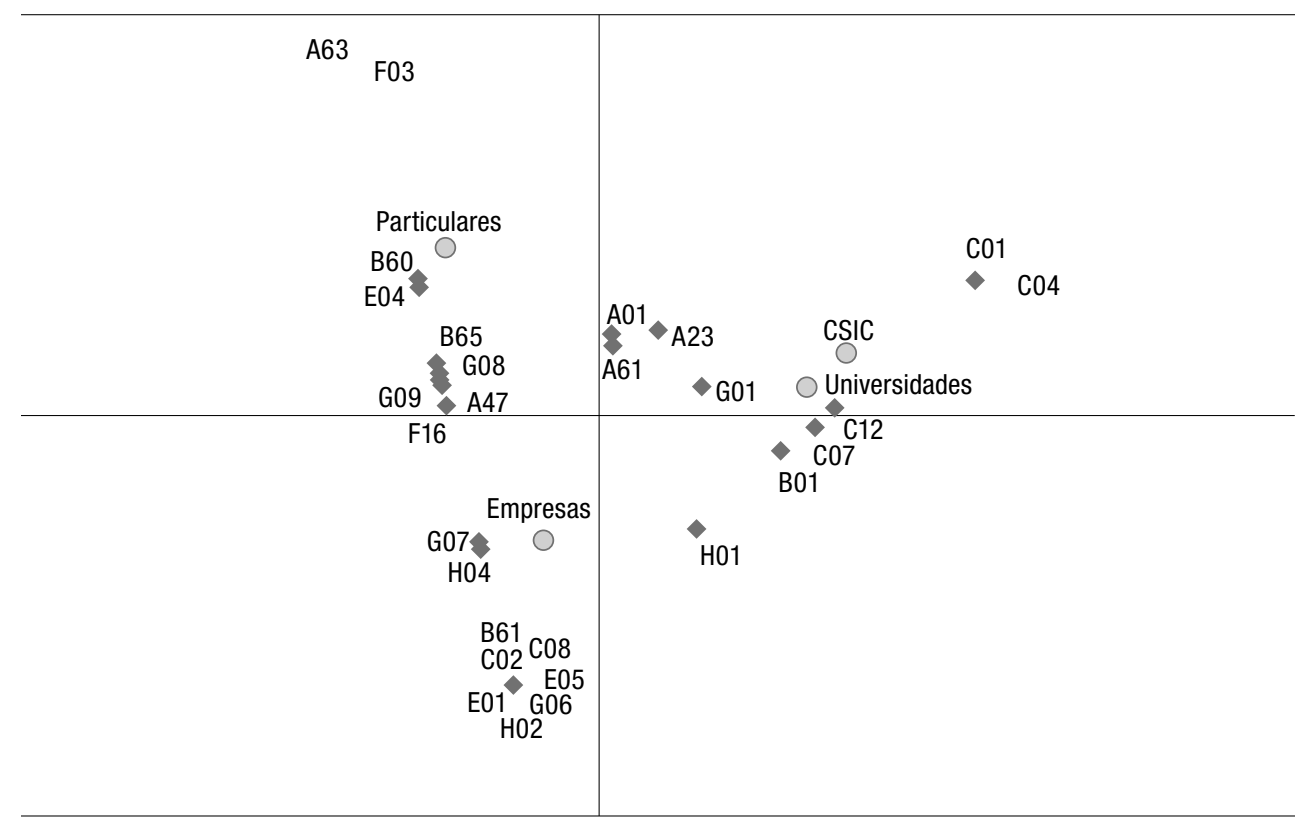

Las frecuencias absolutas de estas clases se presentan en la tabla VIII. A este nivel los temas de mayor producción en nuestro trabajo son las Ciencias Médicas 


\section{TABLA VIII}

Temática de las patentes concedidas a la CM a nivel clase según código CIP

\begin{tabular}{|c|c|c|c|}
\hline & $\begin{array}{l}\text { Cód. } \\
\text { CIP·o }\end{array}$ & Clase & Frec. \\
\hline \multirow{5}{*}{$\mathbf{A}$} & A01 & Agricultura; silvicultura; cría; caza; captura; pesca & 163 \\
\hline & A23 & Alimentos o productos alimenticios; su tratamiento, no cubierto por otras clases & 150 \\
\hline & A 47 & Mobiliario & 142 \\
\hline & A61 & Ciencias médicas o veterinarias; higiene & 435 \\
\hline & A63 & Deportes; juegos; distracciones & 69 \\
\hline \multirow{4}{*}{$\mathbf{B}$} & B01 & Procedimientos o aparatos físicos o químicos en general & 150 \\
\hline & $\mathrm{B} 60$ & Vehículos en general & 166 \\
\hline & B61 & Ferrocarriles & 61 \\
\hline & $\mathrm{B} 65$ & $\begin{array}{l}\text { Transporte; embalaje; almacenado; manipulación de materiales delgados o } \\
\text { filiformes }\end{array}$ & 131 \\
\hline \multirow{6}{*}{ C } & $\mathrm{C} 1$ & Química inorgánica & 61 \\
\hline & $\mathrm{C} 2$ & Tratamiento del agua, agua residual, de alcantarilla o fangos & 78 \\
\hline & $\mathrm{C} 4$ & Cementos: hormigón; piedra artificial; cerámicas; refractarios & 69 \\
\hline & $\mathrm{C} 07$ & Química orgánica & 292 \\
\hline & C08 & $\begin{array}{l}\text { Compuestos macromoleculares orgánicos; su preparación o producción quí- } \\
\text { mica; composiciones basadas en compuestos macromoleculares }\end{array}$ & 72 \\
\hline & C12 & $\begin{array}{l}\text { Bioquímica; cerveza; bebidas alcohólicas; vino; vinagre; microbiología; enzi- } \\
\text { mología; técnicas de mutación o de genética }\end{array}$ & 340 \\
\hline \multirow{3}{*}{$\mathbf{E}$} & E01 & Construcción de carreteras, vías férreas o puentes & 72 \\
\hline & E04 & Edificios & 186 \\
\hline & E05 & Cerraduras; llaves; accesorios de puertas o ventanas; cajas fuertes & 60 \\
\hline \multirow[b]{2}{*}{$\mathbf{F}$} & F03 & Máquinas o motores de líquidos & 42 \\
\hline & F16 & $\begin{array}{l}\text { Elementos o conjuntos de tecnología; medidas generales para asegurar el buen } \\
\text { funcionamiento de las máquinas o instalaciones; aislamiento térmico en general }\end{array}$ & 71 \\
\hline \multirow{5}{*}{ G } & G01 & Metrología & 429 \\
\hline & G06 & Cómputo; cálculo; conteo & 130 \\
\hline & G07 & Dispositivos de control & 155 \\
\hline & G08 & Señalización & 106 \\
\hline & G09 & Enseñanza; criptografía; presentación; publicidad; precintos & 85 \\
\hline \multirow[t]{3}{*}{$\mathbf{H}$} & H01 & $\begin{array}{l}\text { Elementos o conjuntos de tecnología; medidas generales para asegurar el buen } \\
\text { funcionamiento de las máquinas o instalaciones; aislamiento térmico en general }\end{array}$ & 176 \\
\hline & H02 & Producción, conversión o distribución de la energía eléctrica & 96 \\
\hline & H04 & Técnica de las comunicaciones eléctricas & 32 \\
\hline
\end{tabular}

Fuente: OEPM. Elaboración propia. 
o Veterinarias e Higiene, presentes en 435 patentes, seguidos de Metrología en 429, y Bioquímica, Bebidas Alcohólicas, Vinagre, Microbiología, Enzimología, Técnicas de Mutación o Ingeniería Genética en 340 patentes. Estos resultados coinciden con los obtenidos en el Proyecto PIPCYT en relación con las patentes de la CM solicitadas entre los años 2000 y 2007 (Gómez y otros, 2009).

\section{Conclusiones}

Si bien el recuento de patentes no es suficiente para medir la capacidad de innovación de un país, institución o sector productivo, el análisis de sus relaciones con la literatura publicada o de las existentes con los inputs del proceso científico, como son las inversiones en $\mathrm{I}+\mathrm{D}$ y los recursos humanos dedicados a ciencia y tecnología y que aquí se analizan, pueden proporcionar, según determinados autores, información válida sobre la transferencia ciencia-tecnología y sobre la capacidad tecnológica.

Constatamos en primer lugar, tanto el peso de la Comunidad de Madrid en gastos en $\mathrm{I}+\mathrm{D}+\mathrm{i}$, que representa casi el 30\% del total de España en el período estudiado, como el personal dedicado a la investigación, con algo más del 25\% de los investigadores a jornada completa del conjunto nacional.

Estas diferencias se mantienen por sectores de ejecución. Así, la CM realiza comparativamente más inversión en $\mathrm{I}+\mathrm{D}+\mathrm{i}$ en la Administración Pública, por el efecto capitalidad, y en las empresas, mientras que en el conjunto de España, además de en las empresas, es en la Enseñanza Superior donde se ejecuta un mayor gasto. Lo mismo ocurre respecto al número de investigadores, puesto que en el conjunto de España más de la mitad se concentra en la Enseñanza Superior, mientras que en la CM este porcentaje disminuye a la tercera parte.

El sector empresarial manifiesta ejecutar alrededor de la mitad del gasto en I+D+i de todo el conjunto de España, y sin embargo, el número de investigadores que emplea es notablemente inferior, como también ocurre en la CM. Evidentemente, este menor esfuerzo en contratación puede ser uno de los factores que implica el escaso incremento de su actividad patentadora.

Por lo que se refiere a nuestro estudio sobre la capacidad innovadora de la CM, medida a partir del análisis de patentes como indicador de capacidad tecnológica, esta comunidad aportó algo más del 20\% al conjunto nacional durante el período de análisis. Este porcentaje está en consonancia con el obtenido en el estudio mencionado de Gómez y otros (2009), donde observaron que la CM aportó el 19,09\% de las patentes publicadas por la OEPM entre los años 2000 y 2007, siendo esta comunidad tras Cataluña, con el 28,46\% de las patentes, las regiones que presentan una mayor actividad patentadora en el conjunto español. Aunque en nuestro trabajo hemos observado menores incrementos en la evolución de la CM, que permiten pensar que se está produciendo una pérdida de competitividad de la región madrileña frente a otras regiones del estado. 
Dicha actividad patentadora en la CM está vinculada fundamentalmente al mundo empresarial y a los particulares, siendo estos sectores responsables de más del $71 \%$ de las patentes concedidas. Su crecimiento medio anual representa el 11,68\% y el 1,5\% respectivamente.

En el polo opuesto se encuentran los centros públicos de investigación que, con apenas el 26\% de las patentes concedidas en España durante el período analizado, muestran un fuerte incremento de su actividad patentadora en los últimos años del estudio permitiendo prever escenarios cambiantes. Concretamente las universidades incrementan su actividad en más del $28 \%$, mientras que el CSIC lo hace en porcentajes cercanos al 19\%. En este sentido, estos centros son cada vez más conscientes del importante papel que juega la transferencia de conocimiento de la actividad científica que desarrollan.

En cuanto a los temas sobre los que se patenta en la CM, las universidades y CSIC lo hacen fundamentalmente en aquéllos con un fuerte componente investigador, como los vinculados a la Química orgánica e inorgánica. Por su parte, las empresas y particulares muestran un perfil muy diferente, más amplio y difuso, que implica, en la mayoría de los casos, investigación aplicada. Por ejemplo en las empresas, esta actividad se dirige hacia los temas de dispositivos de control y técnica de las comunicaciones eléctricas. También en esta misma línea, las patentes de los particulares se centran en los temas relacionados con vehículos en general, edificios y transportes. Hay que destacar que las patentes relacionadas con la farmacia (A61) están muy centradas en el mapa, por lo que todos los sectores institucionales participan en las mismas.

\section{Bibliografía}

Ahuja, G., y Katila, R. (2001). Technological acquisitions and the innovative performance of acquiring firms: a longitudinal study. Strategic Management Journal, n 21, 197-220.

Arévalo, P. (2005). Una reflexión estratégica sobre la competitividad de las regiones españolas. Análisis Local, n 58 , 33-45.

Audretsch, D. B. (1998). Agglomeration and the location of innovative activity. Oxford Review of Economic Policy, vol. 14 (2), 18-29.

Audretsch, D. B., y Feldman, M. P. (1996). Innovative clusters and the industry life-clycle. Review of Industrial Organization, $\mathrm{n}^{\circ} 11,253-73$.

Balconi, M., et al. (2009). In defence of the linear model: An essay. Research Policy, doi:10.1016/j.respol.2009.09.013.

Buesa, M.; Heijs, J.; Pellitero, M., y Baumert, T. (2006). Regional Systems of innovation and the knowledge production function: the Spanish Case. Technovation, ${ }^{\circ}$ 26, 463-472.

Buesa, M.; Martínez, M.; Heijs, J., y Baumert, T. (2002). Los factores determinantes de la innovación: un análisis econométrico sobre las regiones españolas. Economía Industrial, n 347, 67-84.

Cabrer Borrás, B., y Serrano-Domingo, G. (2007). Innovation and R\&D spillover effects in Spanish regions: A spatial approach. Research Policy, vol. 36 (9), 1357-1371.

Rev. Esp. Doc. Cient., 33, 3, julio-septiembre, 458-479, 2010. ISSN: 0210-0614. doi:10.3989/redc.2010.3.761 
Camacho, J. A., y Rodríguez, M. (2005). Servicios intensivos en conocimiento e innovación regional: un análisis para las regiones europeas, Investigaciones Regionales, $\mathrm{n}^{\circ}$ 7, 91-111.

Comisión Interministerial de Ciencia y Tecnología (200/). Plan Nacional de Investigación Científica, Desarrollo e Innovación Tecnológica 2008-2011. Madrid: Fundación Española para la Ciencia y la Tecnología (FECYT).

Cook, P. (2001). Regional innovation systems, clusters and knowledge economy. Industrial and Corporate Change, vol. 10 (4), 945-974.

Deyle, H. G., y Grupp, H. (2005). Commuters and the regional assignment of innovative activities: a methodological patent study of German districts. Research Policy, vol. 34 (2), 221-234.

Fagerberg, J. (1994). Technology and international differences in growth rate. Journal of Economic Literature, $\mathrm{n}^{\circ}$ 32, 1147-1175.

Feldman, M. P., y Florida, R. (1994). The Geographic Sources of Innovation: Technological Infrastructure and Product Innovation in the United States. Annals of the Association of American Geographers, no 84, 210-229.

Fernández de Lucio, I.; Vega, J., y Gutiérrez Gracia, A. (1983). Estrategias de innovación de las empresas manufactureras españolas. Economistas, año XXVI, n 118, 13-18.

Florida, R. (2005). The flight of the creative class. New York: Harper Collins.

Furman, J. L.; Porter, M. E., y Stern, S. (2002). The determinants of national innovative capacity. Research Policy, n 31, 899-933.

Gómez, I.; Bordons, M.; Morillo, F., y Fernández, M. T. (2005). Regionalization of science and technology data in Spain. Research Evaluation, vol. 14 (2), 137-148.

Gómez, I.; Bordons, M.; Morillo, F., y Moreno, L. (2009). Proyecto de obtención de indicadores de producción científica y tecnológica de la Comunidad de Madrid 2000-2007 (PIPCYT).Madrid: IEDCYT, CSIC.

Grossman, G. M., y Helpman, E. (1991). Innovation and Growth in the Global Economy. Cambridge: MIT Press.

Hagedoon, J., y Cloddt, M. (2003). Measuring innovative performance: is there an advantage in using multiple indicators? Research Policy, vol. 32 (8), 1365-1379.

INEbase. http://www.ine.es/ [consultado el 20 de marzo de 2009].

Krugman, P. (1991). Geography and Trade. Cambridge: Harvard University Press.

Morgan, K., y Nauwelaers, C. (1999). Regional Innovation Strategies: the Challenge for Less-Favoured Regions, London: The Stationery Office and The Regional Studies Association, Taylor and Francis Group.

Narin, F., y Noma, E. (1987). Patents as indicators of corporate technological strength. Research Policy, no 16, 143-155.

Nelson, A. J. (2009). Measuring knowledge spillovers: what patents, licenses and publications reveal about innovation diffusion. Research Policy, vol. 38 (6), 994-1005.

OECD (2005). The New Economy: Beyond the Hyde. Final Report on the OECD Growth Project. Executive Summary. Paris: OECD.

Rodríguez Pose, A., y Crescenzi, R. (2008). Research and Development, Spillovers, Innovations Systems, and the Genesis of Regional Growth in Europe. Regional Studies, vol. 42 (1), 51-67. 
Santos Martín, J. I.; Olmo Martínez, R., y Pajares Gutiérrez, J. (2005). Fenómenos de difusión de conocimiento y dimensión geográfica de la innovación, una aproximación multi-agente. IX Congreso de Ingeniería de Organización. Gijón, 8 y 9 de septiembre.

Trajtenberg, M. (1990). Patents as indicators of innovation. Economic Analysis of Product Innovation. Cambridge: Harvard University Press. 